IJMMS 30:12 (2002) 761-770

PII. S0161171202107150

http://ijmms.hindawi.com

(c) Hindawi Publishing Corp.

\title{
ON COMPOSITION OF FORMAL POWER SERIES
}

\author{
XIAO-XIONG GAN and NATHANIEL KNOX
}

Received 15 July 2001

\begin{abstract}
Given a formal power series $g(x)=b_{0}+b_{1} x+b_{2} x^{2}+\cdots$ and a nonunit $f(x)=a_{1} x+$ $a_{2} x^{2}+\cdots$, it is well known that the composition of $g$ with $f, g(f(x))$, is a formal power series. If the formal power series $f$ above is not a nonunit, that is, the constant term of $f$ is not zero, the existence of the composition $g(f(x))$ has been an open problem for many years. The recent development investigated the radius of convergence of a composed formal power series like $f$ above and obtained some very good results. This note gives a necessary and sufficient condition for the existence of the composition of some formal power series. By means of the theorems established in this note, the existence of the composition of a nonunit formal power series is a special case.
\end{abstract}

2000 Mathematics Subject Classification: 13F25, 13 J05.

1. Introduction and definitions. It is clear that the concepts of power series and formal power series are related but distinct. So we begin with the definition of formal power series.

DEFINITION 1.1. Let $S$ be a ring, let $l \in \mathbb{N}$ be given, a formal power series on $S$ is defined to be a mapping from $\mathbb{N}^{l}$ to $S$, where $\mathbb{N}$ represents the natural numbers. We denote the set of all such mappings by $\mathbb{X}(S)$, or $\mathbb{X}$.

In this note, we only discuss formal power series from $\mathbb{N}$ to $S$. A formal power series $f$ in $x$ from $\mathbb{N}$ to $S$ is usually denoted by

$$
f(x)=a_{0}+a_{1} x+\cdots+a_{n} x^{n}+\cdots, \quad\left\{a_{j}\right\}_{j=0}^{\infty} \subset S .
$$

In this case, $a_{k}, k \in \mathbb{N} \cup\{0\}$ is called the $k$ th coefficient of $f$. If $a_{0}=0, f$ is called a nonunit.

Let $f$ and $g$ be formal power series in $x$ with $f(x)=\sum_{n=0}^{\infty} f_{n} x^{n}$ and $g(x)=$ $\sum_{n=0}^{\infty} g_{n} x^{n}$, and let $r \in S$, then $g+f, r f$, and $g \cdot f$ are defined as

$$
\begin{gathered}
(g+f)(x)=g(x)+f(x)=\sum_{n=0}^{\infty}\left(g_{n}+f_{n}\right) x^{n}, \\
(r f)(x)=r f(x)=\sum_{n=0}^{\infty}\left(r f_{n}\right) x^{n}, \\
(f \cdot g)(x)=g(x) \cdot f(x)=\sum_{n=0}^{\infty} c_{n} x^{n}, \quad c_{n}=\sum_{j=0}^{n} g_{j} f_{n-j}, n=0,1,2, \ldots .
\end{gathered}
$$

It is clear that all those operations are well defined, that is, $g+f, r f$, and $g \cdot f$ are all in $\mathbb{X}$. 
We define the composition of formal power series as follows.

DEFINITION 1.2. Let $S$ be a ring with a metric and let $\mathbb{X}$ be the set of all formal power series over $S$. Let $g \in \mathbb{X}$ be given, say $g(x)=\sum_{k=0}^{\infty} b_{k} x^{k}$. We define a subset $\mathbb{X}_{g} \subset \mathbb{X}$ to be

$$
\mathbb{X}_{g}=\left\{f \in \mathbb{X} \mid f(x)=\sum_{k=0}^{\infty} a_{k} x^{k}, \sum_{n=0}^{\infty} b_{n} a_{k}^{(n)} \in S, k=0,1,2, \ldots\right\},
$$

where $f^{n}(x)=\sum_{k=0}^{\infty} a_{k}^{(n)} x^{k}$, for all $n \in \mathbb{N}$, created by the product rule in Definition 1.1. We will see that $\mathbb{X}_{g} \neq \varnothing$ by Proposition 1.6. Then the mapping $T_{g}: \mathbb{X}_{g} \rightarrow \mathbb{X}$ such that

$$
T_{g}(f)(x)=\sum_{k=0}^{\infty} c_{k} x^{k}
$$

where $c_{k}=\sum_{n=0}^{\infty} b_{n} a_{k}^{(n)}, k=0,1,2, \ldots$, is well defined. We call $T_{g}(f)$ the composition of $g$ and $f ; T_{g}(f)$ is also denoted by $g \circ f$.

Some progress has been made toward determining sufficient conditions for the existence of the composition of formal power series. The most recent development can be found in [1] where Chaumat and Chollet investigated the radius of convergence of composed formal power series and obtained some very good results.

Consider the following examples before going any further.

EXAMPLE 1.3. Let $S=\mathbb{R}$. Let $g(x)=\sum_{n=0}^{\infty} x^{n}$ and $f(x)=1+x$. We cannot calculate even the first coefficient of the series $\sum_{n=0}^{\infty}(f(x))^{n}$ under Definition 1.2. Thus, the composition $g(f(x))$ does not exist.

EXAMPLE 1.4. Let $S=\mathbb{R}, g(x)=\sum_{n=0}^{\infty} x^{n}$, and $f(x)=\sum_{n=1}^{\infty} n ! x^{n}$. It is clear that the series $\sum_{n=1}^{\infty} n ! x^{n}$ converges nowhere except $x=0$. However, one checks that the composition $g(f(x))$, not a composition of functions, is a formal power series.

In Example 1.3 note the difference between the composition of formal power series and the composition of functions such as analytic functions. That is why one is not surprised to read the concern from Henrici [2]. Example 1.4 shows that many convergence results in calculus may not be assumed or applied here.

Some progress has been made toward determining sufficient conditions for the existence of the composition of formal power series.

DEFINITION 1.5. Let $f(x)=\sum_{n=0}^{\infty} a_{n} x^{n}$ be a formal power series. The order of $f$ is the least integer $n$ for which $a_{n} \neq 0$, and denoted by ord $(f)$. The norm, \|\| , of $f$ is defined as $\|f\|=2^{-\operatorname{ord}(f)}$, except that the norm of the zero formal power series is defined to be zero.

Under these definitions, a composition was established as follows.

Proposition 1.6 (see [3]). Let $f(x)=\sum f_{n} x^{n}$ be a formal power series in $x$. If $g$ is a formal power series, such that

$$
\lim _{n \rightarrow \infty}\left\|f_{n} g^{n}\right\|=0,
$$


then the sum $\sum f_{n} g^{n}$ converges to a power series. This series is called the composition of $f$ and $g$ and is denoted by $f \circ g$.

Clearly, the requirement $\lim _{n \rightarrow \infty}\left\|f_{n} g^{n}\right\|=0$ implies that the only candidates for such $g$ are formal power series with constant term equal to zero unless $f$ is a polynomial.

Is this restriction necessary for the existence of the composition of formal power series? What classes of the formal power series can be allowed to participate in the composition? Additionally, is there any sufficient and necessary condition for composition of formal power series? Some of these questions are answered in this note.

2. Coefficients of $f^{n}(x)$. A formal power series is actually the sequence of its coefficients. The composition of formal power series is eventually, or can only be, determined by their coefficients. First, we investigate the coefficients of $f^{n}(x)$ if $f(x)$ is a formal power series. Of course, mathematical induction or the multinomial coefficients can be used to initiate the investigation of the coefficients of $f^{n}(x)$. We show that the $k$ th coefficient of $f^{n}(x)$ mainly depends on $a_{0}$. This property leads to the main theorem.

DEFINITION 2.1. Let $f(x)=a_{0}+a_{1} x+a_{2} x^{2}+\cdots+a_{k} x^{k}+\cdots$ be a formal power series. For every $n \in \mathbb{N}$, we write

$$
f^{n}(x)=a_{0}^{(n)}+a_{1}^{(n)} x+a_{2}^{(n)} x^{2}+\cdots+a_{k}^{(n)} x^{k}+\cdots,
$$

and put $a_{k}^{(1)}=a_{k}$ for all $k \in \mathbb{N} \cup\{0\} ; a_{k}^{(n)}$ is called the $k$ th coefficient of $f^{n}$.

If $a_{m} \neq 0$ but $a_{j}=0$ for all $j>m$, we define the degree of $f$ to be the number $\operatorname{deg}(f)=m$. If there is no such a number $m$, we say that $\operatorname{deg}(f)=\infty$.

Suppose that $S$ is commutative and let $n \in \mathbb{N}$ be given. For any $k \in \mathbb{N} \cup\{0\}$, the $k$ th coefficient $a_{k}^{(n)}$ is determined by the multinomial

$$
\left(a_{0}+a_{1} x+a_{2} x^{2}+\cdots+a_{k} x^{k}\right)^{n}
$$

only, because $a_{t} x^{t}, t>k$, cannot contribute anything to $a_{k}^{(n)}$. Therefore, by the multinomial theorem

$$
a_{k}^{(n)}=\sum\left(\begin{array}{c}
n \\
r_{0}
\end{array}\right)\left(\begin{array}{c}
n-r_{0} \\
r_{1}
\end{array}\right)\left(\begin{array}{c}
n-r_{0}-r_{1} \\
r_{2}
\end{array}\right) \cdots\left(\begin{array}{c}
n-r_{0}-r_{1}-\cdots-r_{k-1} \\
r_{k}
\end{array}\right) a_{0}^{r_{0}} a_{1}^{r_{1}} \cdots a_{k}^{r_{k}},
$$

where the sum is taken for all possible nonnegative integers $r_{0}, r_{1}, \ldots, r_{k}$, such that $r_{0}+r_{1}+\cdots+r_{k}=n$ and $r_{1}+2 r_{2}+3 r_{3}+\cdots+k r_{k}=k$.

For any $n \in \mathbb{N}$ and $k \in \mathbb{N} \cup\{0\}$, we denote

$$
R_{k}^{(n)}=\left\{\mathbf{r} \mid \mathbf{r}=\left(r_{0}, r_{1}, \ldots, r_{k}\right) \in(\mathbb{N} \cup\{0\})^{k+1}, \sum_{j=0}^{k} r_{j}=n, \sum_{j=0}^{k} j r_{j}=k\right\},
$$


and then define

$$
\begin{aligned}
& A_{\mathbf{r}}^{(n)}=\left(\begin{array}{c}
n \\
r_{0}
\end{array}\right)\left(\begin{array}{c}
n-r_{0} \\
r_{1}
\end{array}\right)\left(\begin{array}{c}
n-r_{0}-r_{1} \\
r_{2}
\end{array}\right) \cdots\left(\begin{array}{r}
n-r_{0}-r_{1}-\cdots-r_{k-1} \\
r_{k}
\end{array}\right), \\
& \forall \mathbf{r}=\left(r_{0}, r_{1}, \ldots, r_{k}\right) \in R_{k}^{(n)}, \\
& B_{\mathbf{r}}^{(n)}=\left(\begin{array}{c}
n-r_{0} \\
r_{1}
\end{array}\right)\left(\begin{array}{c}
n-r_{0}-r_{1} \\
r_{2}
\end{array}\right) \cdots\left(\begin{array}{r}
n-r_{0}-r_{1}-\cdots-r_{k-1} \\
r_{k}
\end{array}\right), \\
& \forall \mathbf{r}=\left(r_{0}, r_{1}, \ldots, r_{k}\right) \in R_{k}^{(n)} .
\end{aligned}
$$

Then $A_{\mathbf{r}}^{(n)}=\left(\begin{array}{c}n \\ r_{0}\end{array}\right) B_{\mathbf{r}}^{(n)}$, for all $\mathbf{r} \in R_{k}^{(n)}$ and

$$
\begin{aligned}
a_{k}^{(n)} & =\sum_{\mathbf{r} \in R_{k}^{(n)}} A_{\mathbf{r}}^{(n)} a_{0}^{r_{0}} a_{1}^{r_{1}} a_{2}^{r_{2}} \cdots a_{k}^{r_{k}} \\
& =\sum_{\mathbf{r} \in R_{k}^{(n)}}\left(\begin{array}{c}
n \\
r_{0}
\end{array}\right) B_{\mathbf{r}} a_{0}^{r_{0}} a_{1}^{r_{1}} a_{2}^{r_{2}} \cdots a_{k}^{r_{k}} .
\end{aligned}
$$

For any $n \in \mathbb{N}$ and $k \in \mathbb{N} \cup\{0\}$, since $\sum_{j=1}^{k} j r_{j}=\sum_{j=0}^{k} j r_{j}=k$ for every $\left(r_{0}, r_{1}, \ldots, r_{k}\right)$ $\in R_{k}^{(n)}$, the number of selections of the $k$-tuple $\left(r_{1}, r_{2}, \ldots, r_{k}\right)$ is finite, no matter how large $n$ is. This property, which will be proved in Lemma 2.2, is very important for the investigation of $a_{k}^{(n)}$.

LEMMA 2.2. Let $k, m \in \mathbb{N} \cup\{0\}$ be given. Then

(i) $\left(r_{0}, r_{1}, \ldots, r_{k}\right) \in R_{k}^{(k+m)} \Rightarrow r_{0} \geq m$,

(ii) $\left(r_{0}, r_{1}, \ldots, r_{k}\right) \in R_{k}^{(k)} \Leftrightarrow\left(r_{0}+m, r_{1}, \ldots, r_{k}\right) \in R_{k}^{(k+m)}$,

(iii) $\left|R_{k}^{(k)}\right|=\left|R_{k}^{(k+m)}\right|$,

where $|V|$ denotes the cardinal number of the set $V$.

PRoof. $\left(r_{0}, r_{1}, \ldots, r_{k}\right) \in R_{k}^{(k+m)} \Rightarrow \sum_{j=0}^{k} r_{j}=k+m$ and $\sum_{j=0}^{k} j r_{j}=k$. Then,

$$
r_{0}=k+m-\sum_{j=1}^{k} r_{j} \geq k+m-\sum_{j=1}^{k} j r_{j}=m .
$$

This is (i).

Next, $\left(r_{0}, r_{1}, \ldots, r_{k}\right) \in R_{k}^{(k)} \Rightarrow \sum_{j=0}^{k} r_{j}=k$ and $\sum_{j=0}^{k} j r_{j}=k \Rightarrow r_{0}+m+\sum_{j=1}^{k} r_{j}=k+m$ and $0\left(r_{0}+m\right)+\sum_{j=1}^{k} j r_{j}=k \Rightarrow\left(r_{0}+m, r_{1}, \ldots, r_{k}\right) \in R_{k}^{(k+m)}$. This proves the necessity of (ii).

Let $\left(r_{0}, r_{1}, \ldots, r_{k}\right) \in R_{k}^{(k+m)}$ be given. Then $\sum_{j=0}^{k} r_{j}=k+m$ and $\sum_{j=0}^{k} j r_{j}=k$. Then (i) yields that $r_{0} \geq m$, and then $r_{0}-m \in \mathbb{N} \cup\{0\}$. Then we have

$$
\left(r_{0}-m, r_{1}, \ldots, r_{k}\right) \in R_{k}^{(k)}
$$

because $\left(r_{0}-m\right)+\sum_{j=1}^{k} r_{j}=k$ and $0 \cdot\left(r_{0}-m\right)+\sum_{j=1}^{k} j r_{j}=k$. This is the sufficiency of (ii). Thus we have proved (ii).

By (ii), the mapping $\left(r_{0}, r_{1}, \ldots, r_{k}\right) \rightarrow\left(r_{0}+m, r_{1}, \ldots, r_{k}\right)$ from every $\left(r_{0}, r_{1}, \ldots, r_{k}\right) \in$ $R_{k}^{(k)}$ to $\left(r_{0}+m, r_{1}, \ldots, r_{k}\right) \in R_{k}^{(k+m)}$ is well defined and the mapping is obviously oneto-one, which proves (iii).

The proof is completed. 
Lemma 2.2 gives some significant properties of the coefficients of a formal power series. We use the next corollary to point out these important results.

COROllary 2.3. Let $k, m \in \mathbb{N} \cup\{0\}$ be given. Let $f$ be a formal power series as in Definition 1.1 and let $S$ be a commutative ring. Then, by (2.7), in the expressions

$$
\begin{aligned}
a_{k}^{(k)} & =\sum_{\mathbf{r} \in R_{k}^{(k)}} A_{\mathbf{r}}^{(k)} a_{0}^{r_{0}} a_{1}^{r_{1}} \cdots a_{k}^{r_{k}}, \\
a_{k}^{(k+m)} & =\sum_{\mathbf{q} \in R_{k}^{(k+m)}} A_{\mathbf{q}}^{(k+m)} a_{0}^{q_{0}} a_{1}^{q_{1}} \cdots a_{k}^{q_{k}},
\end{aligned}
$$

the sums have the same number of summands. The number of summands is determined by $k$ only. The number of terms in these two sums are the same, the coefficients, $A_{\mathrm{r}}^{(k)}$ 's, of terms and the power of $a_{0}$ may be different.

To find the relationship between $A_{\left(r_{0}+m, r_{1}, \ldots, r_{k}\right)}^{(k+m)}$ and $A_{\left(r_{0}, r_{1}, \ldots, r_{k}\right)}^{(k)}$, like $A_{\mathbf{r}}^{(k)}$ and $A_{\mathbf{q}}^{(k+m)}$ in Corollary 2.3 explicitly, for any $k, m \in \mathbb{N} \cup\{0\}$ and $\mathbf{r}=\left(r_{0}, r_{1}, \ldots, r_{k}\right) \in R_{k}^{(k)}$, only apply Lemma 2.2(ii) and (2.7) to the second form in Corollary 2.3. This relationship can be described in the following corollary.

COROLlaRY 2.4. Let $k, m \in \mathbb{N} \cup\{0\}$ be given. Let $f$ be a formal power series as in Definition 1.1. If $S$ is commutative and $\mathbf{r}=\left(r_{0}, r_{1}, \ldots, r_{k}\right) \in R_{k}^{(k)}$, then

$$
\begin{gathered}
A_{\left(r_{0}+m, r_{1}, \ldots, r_{k}\right)}^{(k+m)}=\left(\begin{array}{c}
k+m \\
k-r_{0}
\end{array}\right) B_{\left(r_{0}, \ldots, r_{k}\right)}^{(k)}, \\
a_{k}^{(k+m)}=\sum_{\mathbf{r} \in R_{k}^{(k)}}\left(\begin{array}{l}
k+m \\
k-r_{0}
\end{array}\right) B_{\mathbf{r}}^{(k)} a_{0}^{r_{0}+m} a_{1}^{r_{1}} \cdots a_{k}^{r_{k}},
\end{gathered}
$$

where $B_{\mathbf{r}}^{(k)}$ is defined as in (2.6).

Definition 2.5. Let $n \in \mathbb{N}$ and $k \in \mathbb{N} \cup\{0\}$ be given. If $\mathbf{r}=\left(r_{0}, r_{1}, \ldots, r_{k}\right) \in R_{k}^{(n)}$, denote that

$$
R(s)_{k}^{(n)}=\left\{\mathbf{r} \in R_{k}^{(n)} \mid \mathbf{r}=\left(r_{0}, 0, \ldots, 0, r_{s}, r_{s+1}, \ldots, r_{k}\right)\right\}, \quad \forall 1 \leq s \leq k .
$$

It is obvious that, $R(s)_{k}^{(n)} \subset R_{k}^{(n)}$.

LEMMA 2.6. Let $k \in \mathbb{N} \cup\{0\}$ and $s \in \mathbb{N}$ be given and let

$$
\mathbf{r}(s)=(k s-k, 0, \ldots, 0, k, 0, \ldots, 0) \in(\mathbb{N} \cup\{0\})^{k s},
$$

where the sth coordinate of $\mathbf{r}(s)$ is $k$ and the other coordinates of $\mathbf{r}(s)$ are zero except the 0's. Then

(i) $\mathbf{r}(s) \in R(s)_{k s}^{(k s)}$;

(ii) if $\mathbf{r}=\left(r_{0}, 0, \ldots, 0, r_{s}, r_{s+1}, \ldots, r_{k s}\right) \in R(s)_{k s}^{(k s)}$, then $r_{0} \geq k(s-1)$;

(iii) in (ii), $r_{0}=k(s-1) \Leftrightarrow \mathbf{r}=\mathbf{r}(s)$.

Proof. For $\mathbf{r}(s), k(s-1)+k=k s$ and $k \cdot s=k s$ imply that $\mathbf{r}(s) \in R_{k s}^{(k s)}$. Then (i) follows from Definition 2.5. 


$$
\begin{gathered}
\text { Next, } \mathbf{r}=\left(r_{0}, 0, \ldots, 0, r_{s}, r_{s+1}, \ldots, r_{k s}\right) \in R(s)_{k s}^{(k s)} \subset R_{k s}^{(k s)} \text { implies that } \\
r_{0}+r_{s}+r_{s+1}+\cdots+r_{k s}=k s, \\
s r_{s}+(s+1) r_{s+1}+\cdots+k s r_{k s}=k s .
\end{gathered}
$$

Then, $k=r_{s}+((s+1) / s) r_{s+1}+((s+2) / s) r_{s+2}+\cdots+k r_{k s}$. Since $r_{j} \geq 0$ for all $j$, it follows that

$$
k \geq r_{s}+r_{s+1}+\cdots+r_{k s} .
$$

Then $r_{0}=k s-\sum_{j=s}^{k s} r_{j} \geq k s-k=k(s-1)$. This is (ii).

Finally, we show (iii). The sufficiency is obvious, we need only show necessity. Suppose that $r_{0}=k(s-1)$. Then,

$$
\begin{gathered}
r_{s}+r_{s+1}+\cdots+r_{k s}=k s-k(s-1)=k, \\
r_{s}+\frac{s+1}{s} r_{s+1}+\cdots+k r_{k s}=k .
\end{gathered}
$$

Notice that $r_{j} \geq 0$ for all $j, s \leq j \leq k s$. We have

$$
r_{j}=0, \quad j=s+1, s+2, \ldots, s k .
$$

Then $r_{s}=k s-r_{0}=k$, and hence $\mathbf{r}=\mathbf{r}(s)$.

The proof is completed.

3. Composition of formal power series. A formal power series is a mapping from $\mathbb{N}$ to a ring $S$. If this ring is endowed with a metric, the pointwise convergence of a mapping from the set of formal power series to itself is well defined. This gives us a way to define a composition in the set of formal power series over a ring.

THEOREM 3.1. Let $S$ be a field with a metric, let $\mathbb{X}$ be the set of all formal power series from $\mathbb{N}$ to $S$, and let $f, g \in \mathbb{X}$ be given with the forms

$$
\begin{aligned}
& f(x)=a_{0}+a_{1} x+\cdots+a_{n} x^{n}+\cdots, \\
& g(x)=b_{0}+b_{1} x+\cdots+b_{n} x^{n} \cdots,
\end{aligned}
$$

and $\operatorname{deg}(f) \neq 0$. Then, the composition $g \circ f$ exists if and only if

$$
\sum_{n=k}^{\infty}\left(\begin{array}{l}
n \\
k
\end{array}\right) b_{n} a_{0}^{n-k} \in S, \quad \forall k \in \mathbb{N} \cup\{0\} .
$$

Proof. Suppose that $g \circ f$ exists, that is, $c_{k}=\sum_{n=0}^{\infty} b_{n} a_{k}^{(n)}$ exists for all $k$, or, the series $\sum_{n=0}^{\infty} b_{n} a_{k}^{(n)}$ converges in $S$ for all $k$. We show that (3.2) is true by mathematical induction on $k$. Since the conclusion is obvious if $a_{0}=0$, assume that $a_{0} \neq 0$.

It is clear that (3.2) is true for $k=0$ because the expression in (3.2) is $c_{0}$. Suppose that (3.2) holds for all $j$ with $0 \leq j \leq k$ for some $k \geq 0$.

Since $\operatorname{deg}(f) \neq 0$, we may find $s \in \mathbb{N}$ such that $1 \leq s \leq \operatorname{deg}(f), a_{s} \neq 0$ but $a_{j}=0$ for all $1 \leq j<s$, that is, $a_{s}$ is the first nonzero coefficient of $f$ except the constant term. Consider $a_{k s+s}^{(n)}$ for any $n \in \mathbb{N}$ with $n \geq(k+1) s$. As in Lemma 2.6, denote $\mathbf{r}(s) \in$ $R(s)_{k s+s}^{(k s+s)}$ by

$$
\mathbf{r}(s)=((k+1) s-(k+1), 0, \ldots, 0, k+1,0, \ldots, 0),
$$


where the $s$ th coordinate of $\mathbf{r}(s)$ is $k+1$ and the other coordinates are zero except the 0 's. By the second formula in (2.11),

$$
\begin{aligned}
a_{k s+s}^{(n)} & =\sum_{\mathbf{r} \in R_{k s+s}^{(k s+s)}}\left(\begin{array}{c}
n \\
k s+s-r_{0}
\end{array}\right) B_{\mathbf{r}}^{(k s+s)} a_{0}^{n+r_{0}-(k+1) s} a_{1}^{r_{1}} \cdots a_{k s+s}^{r_{k s+s}} \\
& =\sum_{\mathbf{r} \in R(s) k_{k s+s}^{(k s+s)}}\left(\begin{array}{c}
n \\
k s+s-r_{0}
\end{array}\right) B_{\mathbf{r}}^{(k s+s)} a_{0}^{n+r_{0}-(k+1) s} a_{s}^{r_{s}} a_{s+1}^{r_{s+1}} \cdots a_{k s+s}^{r_{k s+s}}
\end{aligned}
$$

because $a_{1}=a_{2}=\cdots=a_{s-1}=0$. By Lemma 2.6 for $(k+1) s$, we have

$$
\begin{aligned}
a_{k s+s}^{(n)}= & \sum_{\mathbf{r} \in R(s))_{k s+s}^{(k s+s)}, \mathbf{r} \neq \mathbf{r}(s)}\left(\begin{array}{c}
n \\
k s+s-r_{0}
\end{array}\right) B_{\mathbf{r}}^{(k s+s)} a_{0}^{n+r_{0}-(k+1) s} a_{s}^{r_{s}} \cdots a_{k s+s}^{r_{k s+s}} \\
& +\left(\begin{array}{c}
n \\
k s+s-(k s+s)+(k+1)
\end{array}\right) B_{\mathbf{r}(s)}^{(k s+s)} a_{0}^{n+(k+1) s-(k+1)-k s-s} a_{s}^{k+1} \\
= & \sum_{\mathbf{r} \in R(s)_{k s+s}^{(k s+s)}, \mathbf{r} \neq \mathbf{r}(s)}\left(\begin{array}{c}
n \\
k s+s-r_{0}
\end{array}\right) B_{\mathbf{r}}^{(k s+s)} a_{0}^{n+r_{0}-(k+1) s} a_{s}^{r_{s}} \cdots a_{k s+s}^{r_{(k+1) s}} \\
& +\left(\begin{array}{c}
n \\
k+1
\end{array}\right) B_{\mathbf{r}(s)}^{(k s+s)} a_{0}^{n-k-1} a_{s}^{k+1} .
\end{aligned}
$$

Then,

$$
\begin{aligned}
& c_{k s+s}=\sum_{n=0}^{\infty} b_{n} a_{k s+s}^{(n)} \\
& =\sum_{n=k s+s}^{\infty} b_{n} a_{k s+s}^{(n)}+\sum_{n=0}^{k s+s-1} b_{n} a_{k s+s}^{(n)} \\
& =\sum_{n=k s+s}^{\infty} b_{n}\left(\sum_{\mathbf{r} \in R(s)_{k s+s}^{(k s+s)}, \mathbf{r} \neq \mathbf{r}(s)}\left(\begin{array}{c}
n \\
k s+s-r_{0}
\end{array}\right) B_{\mathbf{r}}^{(k s+s)} a_{0}^{n+r_{0}-(k+1) s} a_{s}^{r_{s}} \cdots a_{k s+s}^{r_{k s+s}}\right) \\
& +\sum_{n=k s+s}^{\infty} b_{n}\left(\begin{array}{c}
n \\
k+1
\end{array}\right) B_{\mathbf{r}(s)}^{(k s+s)} a_{0}^{n-k-1} a_{s}^{k+1}+\sum_{n=0}^{k s+s-1} b_{n} a_{k s+s}^{(n)} .
\end{aligned}
$$

We may only consider those $\mathbf{r} \in R(s)_{k s+s}^{(k s+s)}$ for which $a_{s}^{r_{s}} a_{s+1}^{r_{s+1}} \cdots a_{k s+s}^{r_{k s+s}} \neq 0$ in the above expression, and we denote them as $\mathbf{r}^{\prime}=\left(r_{0}^{\prime}, 0, \ldots, 0, r_{s}^{\prime}, \ldots, r_{k s+s}^{\prime}\right) \in R(s)_{k s+s}^{(k s+s)}$. Since $S$ is a field, we have

$$
\begin{aligned}
c_{k s+s}= & \sum_{\mathbf{r}^{\prime} \in R(s)} B_{k s+s}^{(k s)}, \mathbf{r}^{\prime} \neq \mathbf{r}(s) \\
& +B_{\mathbf{r}(s)}^{(k s+s)} a_{s}^{k+1} \sum_{n=k s+s}^{\infty}\left(\begin{array}{c}
n \\
k+1
\end{array}\right) b_{n} a_{0}^{n-k-1}+\sum_{n=0}^{r_{s}^{\prime}} a_{s+1}^{r_{s+1}^{\prime}} \cdots a_{k s+s}^{r_{k s+s}^{\prime}}\left(\sum_{n=k s+s}^{\infty} b_{n} a_{k s+s}^{(n)} .\right.
\end{aligned}
$$

By Lemma 2.6, $\mathbf{r}^{\prime} \neq \mathbf{r}(s)$ in $R(s)_{k s+s}^{(k s+s)} \Rightarrow r_{0}^{\prime}>(k+1) s-(k+1) \Rightarrow k s+s-r_{0}^{\prime}<k+1$. 
Then, $\sum_{n=0}^{\infty}\left(\begin{array}{c}n \\ k s+s-r_{0}^{\prime}\end{array}\right) b_{n} a_{0}^{n+r_{0}^{\prime}-k s-s}$ converges by the inductive hypothesis. Then,

$$
\begin{aligned}
& B_{\mathbf{r}(s)}^{(k s+s)} a_{s}^{k+1} \sum_{n=k s+s}^{\infty}\left(\begin{array}{c}
n \\
k+1
\end{array}\right) b_{n} a_{0}^{n-k-1} \\
& =c_{k s+s}-\sum_{n=0}^{k s+s-1} b_{n} a_{k s+s}^{(n)} \\
& \quad-\sum_{\mathbf{r}^{\prime} \in R_{k s+s}^{(k s+s)}(s, *), \mathbf{r}^{\prime} \neq s} B_{\mathbf{r}^{\prime}}^{(k s+s)} a_{s}^{r_{s}^{\prime}} \cdots a_{k s+s}^{r_{k s+s}^{\prime}}\left(\sum_{n=k s+s}^{\infty}\left(\begin{array}{c}
n \\
k s+s-r_{0}^{\prime}
\end{array}\right) b_{n} a_{0}^{n+r_{0}^{\prime}-k s-s}\right),
\end{aligned}
$$

and the right-hand side converges. Notice that $S$ is a field and $a_{s} \neq 0$, we have (3.2) for $k+1$ and hence we have proved the necessity.

Now suppose that $\sum_{n=k}^{\infty}\left(\begin{array}{l}n \\ k\end{array}\right) b_{n} a_{0}^{n-k}$ converges for every $k \in \mathbb{N} \cup\{0\}$. Let $k \in \mathbb{N} \cup\{0\}$ be given. Consider

$$
c_{k}=\sum_{n=0}^{\infty} b_{n} a_{k}^{(n)} .
$$

By (2.11), with $n=k+m$,

$$
a_{k}^{(n)}=\sum_{\left(r_{0}, \ldots, r_{k}\right) \in R_{k}^{(k)}}\left(\begin{array}{c}
n \\
k-r_{0}
\end{array}\right) B_{\left(r_{0}, \ldots, r_{k}\right)}^{(k)} a_{0}^{n+r_{0}-k} a_{1}^{r_{1}} \cdots a_{k}^{r_{k}},
$$

and the sum is finite. Then,

$$
\begin{aligned}
& c_{k}=\sum_{n=0}^{\infty} b_{n} a_{k}^{(n)}=\sum_{n=0}^{k-1} b_{n} a_{k}^{(n)}+\sum_{n=k}^{\infty} b_{n} a_{k}^{(n)} \\
& =\sum_{n=0}^{k-1} b_{n} a_{k}^{(n)}+\sum_{n=k}^{\infty} b_{n}\left(\sum_{\left(r_{0}, \ldots, r_{k}\right) \in R_{k}^{(k)}}\left(\begin{array}{c}
n \\
k-r_{0}
\end{array}\right) B_{\left(r_{0}, \ldots, r_{k}\right)}^{(k)} a_{0}^{n+r_{0}-k} a_{1}^{r_{1}} \cdots a_{k}^{r_{k}}\right) \text {. }
\end{aligned}
$$

If $a_{0}=0$, then $n>k-r_{0}$ implies that $a_{0}^{n+r_{0}-k}=0$, and hence the above series is a finite sum. Then $c_{k}$ exists, and then the conclusion is true. Now we assume that $a_{0} \neq 0$. Since $\operatorname{deg}(f) \neq 0$, we may only consider those $\mathbf{r} \in R_{k}^{(k)}$ for which $a_{1}^{r_{1}} a_{2}^{r_{2}} \cdots a_{k}^{r_{k}} \neq 0$ in the above expression, and denote them as $\mathbf{r}^{\prime}=\left(r_{0}^{\prime}, r_{1}^{\prime}, \ldots, r_{k}^{\prime}\right)$. Then

$$
\begin{aligned}
c_{k} & =\sum_{n=0}^{k-1} b_{n} a_{k}^{(n)}+\sum_{n=k}^{\infty} b_{n}\left(\sum_{\left(r_{0}^{\prime}, \ldots, r_{k}^{\prime}\right) \in R_{k}^{(k)}}\left(\begin{array}{c}
n \\
k-r_{0}^{\prime}
\end{array}\right) B_{\left(r_{0}^{\prime}, \ldots, r_{k}^{\prime}\right)}^{(k)} a_{0}^{n+r_{0}^{\prime}-k} a_{1}^{r_{1}^{\prime}} \cdots a_{k}^{r_{k}^{\prime}}\right) \\
& =\sum_{n=0}^{k-1} b_{n} a_{k}^{(n)}+\sum_{\mathbf{r}^{\prime} \in R_{k}^{(k)}} B_{\mathbf{r}^{\prime}}^{(k)} a_{1}^{r_{1}^{\prime}} \cdots a_{k}^{r_{k}^{\prime}}\left(\sum_{n=k}^{\infty} b_{n}\left(\begin{array}{c}
n \\
k-r_{0}^{\prime}
\end{array}\right) a_{0}^{n+r_{0}^{\prime}-k}\right)
\end{aligned}
$$

because $S$ is a field. Thus, $c_{k}$ exists in $S$ by (3.2), and we have completed the proof.

REMARK 3.2. If $a_{0}=0$, then $f$ is allowed to be in the composition by Theorem 3.1. If $g$ is a polynomial, then (3.2) is true clearly. These results show that Proposition 1.6 is just a special case of Theorem 3.1. 
THEOREM 3.3. Let $\mathbb{X}$ be the set of all formal power series from $\mathbb{N}$ to the set of complex numbers $\mathbb{C}$. Let $f, g \in \mathbb{X}$ be given with the forms

$$
f(x)=a_{0}+a_{1} x+\cdots+a_{n} x^{n}+\cdots, \quad g(x)=b_{0}+b_{1} x+\cdots+b_{n} x^{n}+\cdots .
$$

If the series $\sum_{n=0}^{\infty} b_{n} R^{n}$ converges for some $R>\left|a_{0}\right|$, then $g \circ f$ exists.

Proof. If $\operatorname{deg}(f)=0$, the conclusion is obvious, so assume that $\operatorname{deg}(f) \neq 0$.

Consider the power series $\sum_{n=0}^{\infty} b_{n} x^{n}$. Since $\sum_{n=0}^{\infty} b_{n} R^{n}$ converges, it follows that the $k$ th derivative

$$
\sum_{n=k}^{\infty} n(n-1) \cdots(n-k+1) b_{n} x^{n-k}
$$

converges for $|x|<R$ for any $k \in \mathbb{N} \cup\{0\}$. Then, for any $k \in \mathbb{N} \cup\{0\}$ given,

$$
\begin{aligned}
\sum_{n=k}^{\infty}\left(\begin{array}{l}
n \\
k
\end{array}\right) b_{n} x^{n-k} & =\sum_{n=k}^{\infty} \frac{n(n-1) \cdots(n-k+1)}{k !} b_{n} x^{n-k} \\
& =\frac{1}{k !} \sum_{n=k}^{\infty} n(n-1) \cdots(n-k+1) b_{n} x^{n-k}
\end{aligned}
$$

converges for $|x|<R$. Then, $\left|a_{0}\right|<R$ implies that

$$
\sum_{n=k}^{\infty}\left(\begin{array}{l}
n \\
k
\end{array}\right) b_{n} a_{0}^{n-k}
$$

converges for all $k \in \mathbb{N} \cup\{0\}$. Then Theorem 3.1 yields the conclusion.

REMARK 3.4. Let $f(x)=a_{0}+a_{1} x+a_{2} x^{2}+\cdots \in \mathbb{X}(\mathbb{R})$ be given. Define the derivative of $f$ to be the formal power series

$$
f^{\prime}(x)=a_{1}+a_{2} x+a_{3} x^{2}+\cdots
$$

Then (3.2) is equivalent to

$$
g^{(k)}\left(a_{0}\right) \in S, \quad \forall k \in \mathbb{N} \cup\{0\}
$$

by the proof of Theorem 3.3. However, Example 1.4 tells us how careful we have to be when we try to assume any result from calculus.

COROLlary 3.5. Let $f, g \in \mathbb{X}(\mathbb{C})$ be given by

$$
f(x)=a_{0}+a_{1} x+\cdots+a_{n} x^{n}+\cdots, \quad g(x)=b_{0}+b_{1} x+\cdots+b_{n} x^{n}+\cdots .
$$

Suppose that $\left|a_{0}\right|<1$ and $\left|b_{n}\right| \leq M$, for all $n \in \mathbb{N}$ for some positive number $M$. Then $g \circ f$ is well defined.

Proof. Pick $R$ such that $\left|a_{0}\right|<R<1$, then $\sum_{n=0}^{\infty} b_{n} R^{n}$ converges because

$$
\left|b_{n}\right| \leq M, \quad \forall n \in \mathbb{N}
$$

and $\sum_{n=0}^{\infty} R^{n}$ converges. Applying Theorem 3.3, $g \circ f$ exists. 
EXAMPLE 3.6. Let $S=\mathbb{R}, g(x)=\sum_{n=0}^{\infty} x^{n}$, and $f(x)=0.5+\sum_{n=1}^{\infty} n ! x^{n}$. Corollary 3.5 of Theorem 3.3 yields that $g \circ f$ exists.

Theorems 3.1 and 3.3 tell us that the existence of $g \circ f$ strongly depends on the constant term of $f$ and the coefficients of $g$. This result directs us to a deeper investigation of the subset of $\mathbb{X}$ in which the composition is closed. It is clear that Theorems 3.1 and 3.3 can be applied to ordinary power series.

\section{REFERENCES}

[1] J. Chaumat and A.-M. Chollet, On composite formal power series, Trans. Amer. Math. Soc. 353 (2001), no. 4, 1691-1703.

[2] P. Henrici, Applied and Computational Complex Analysis. Vol. 1. Power SeriesIntegration-Conformal Mapping-Location of Zeros, John Wiley \& Sons, New York, 1988.

[3] S. Roman, Advanced Linear Algebra, Graduate Texts in Mathematics, vol. 135, SpringerVerlag, New York, 1992.

Xiao-Xiong Gan and Nathaniel Knox: Department of Mathematics, Morgan State UNIVERSITY, BALTIMORE, MD 21251, USA 


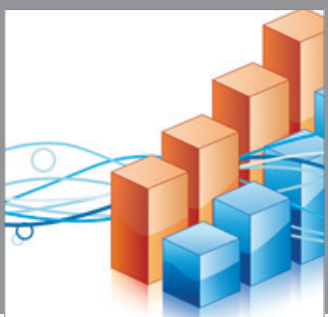

Advances in

Operations Research

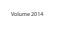

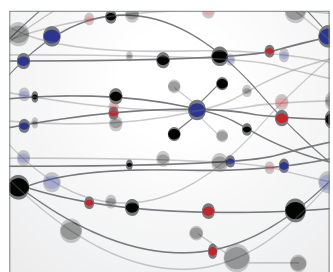

\section{The Scientific} World Journal
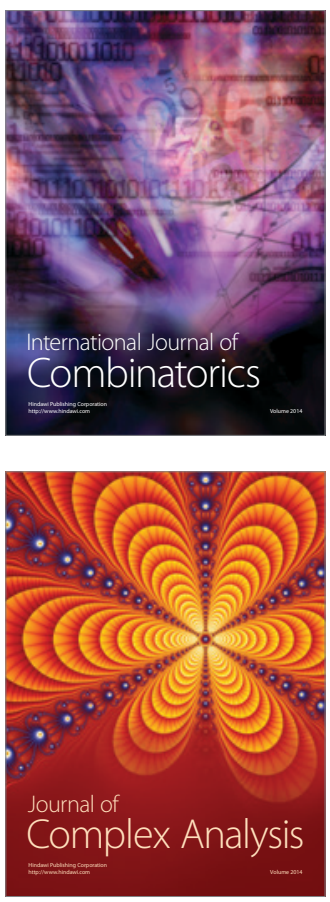

International Journal of

Mathematics and

Mathematical

Sciences
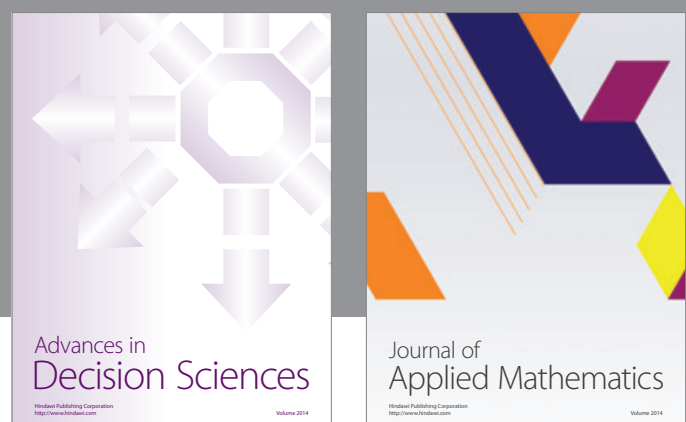

Journal of

Applied Mathematics
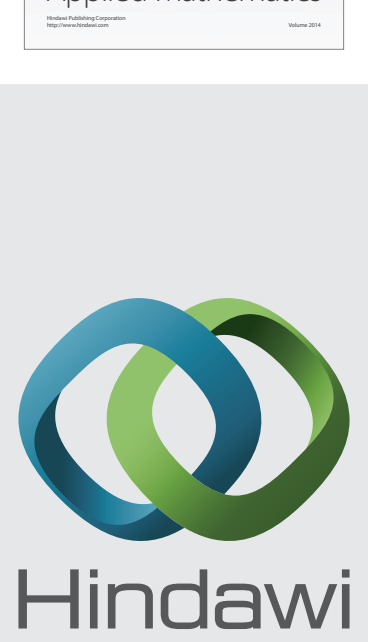

Submit your manuscripts at http://www.hindawi.com
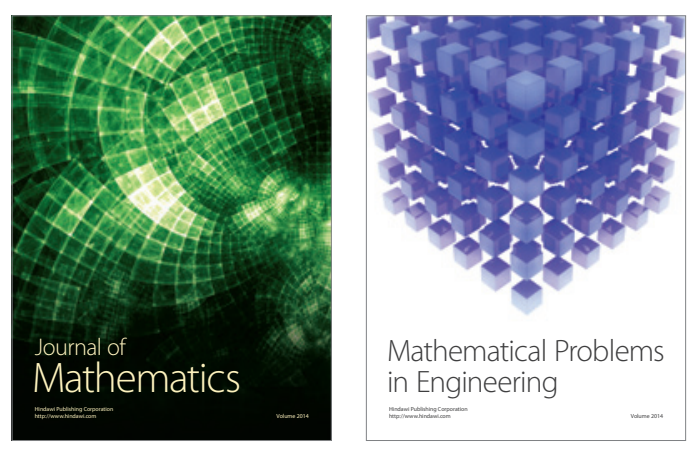

Mathematical Problems in Engineering
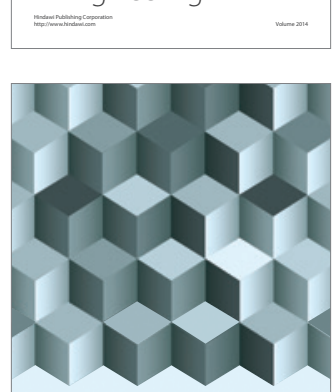

Journal of

Function Spaces
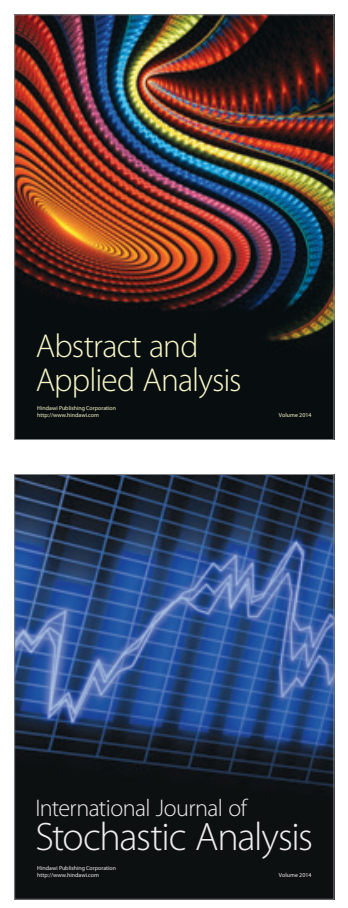

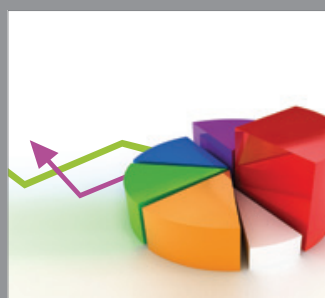

ournal of

Probability and Statistics

Promensencen
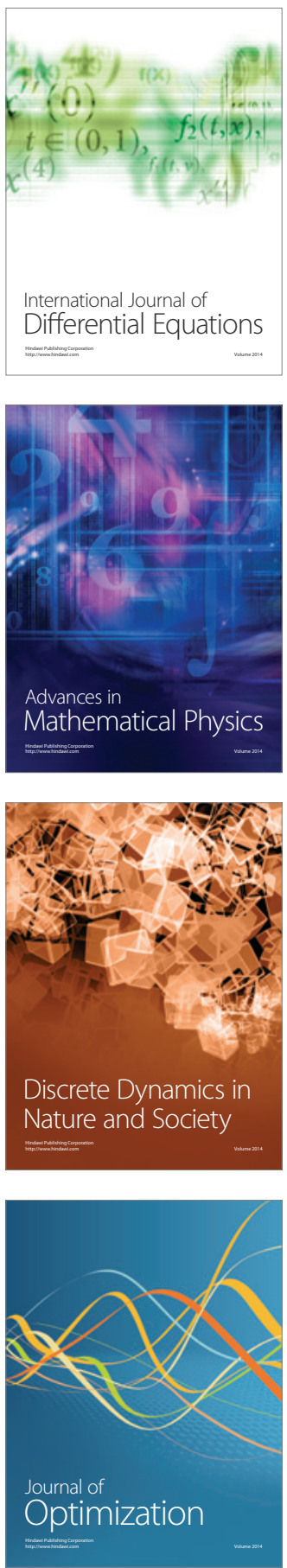